\title{
Genetic Recombination of Human Immunodeficiency Virus
}

\author{
FRANÇOIS CLAVEL, ${ }^{*}$ M. DAVID HOGGAN, RONALD L. WILLEY, KLAUS STREBEL, \\ MALCOLM A. MARTIN, AND ROY REPASKE \\ Laboratory of Molecular Microbiology, National Institute of Allergy and Infectious Diseases, Bethesda, Maryland 20892
}

Received 7 September 1988/Accepted 11 November 1988

\begin{abstract}
We investigated genetic recombination of the human immunodeficiency virus (HIV) in a tissue culture system. A clonal cell line expressing a single integrated HIV provirus with a termination codon affecting pol gene expression was transfected with different defective mutants derived from an infectious molecular clone of HIV. Replication-competent viral particles were recovered, passaged, and plaque purified. Restriction analyses of the proviral DNA corresponding to several of these viruses indicated that their emergence was the result of genetic recombination.
\end{abstract}

Genetic recombination is a frequently observed feature of the retrovirus life cycle. High-frequency exchange of genetic determinants has been reported for both avian and murine type $\mathrm{C}$ viruses $(1,5,6,14-18,26,27,30)$. Although recombination between lentiviruses has not been widely documented, it must be assumed to occur and to contribute to the genetic variability characteristic of this retrovirus subfamily. The variability of the human immunodeficiency virus (HIV) genome has been particularly well documented $(3,23,28)$ and could result in part from recombinational events in addition to mutations affecting individual nucleotides. Another characteristic of HIV infection in humans is its chronicity and slow progression toward immunodeficiency. HIV genomes containing mutations that compromise efficient replication could contribute to this chronicity. Their repair through recombinational mechanisms could result in HIV disease progression.

We previously described (10) a cloned cell line (8E5) that was isolated from a mass culture of A3.01 cells (9) surviving acute HIV infection. These cells contain a single copy of a defective HIV provirus with a mutation (1-base-pair deletion) in the pol gene that precludes the synthesis of reverse transcriptase (RT) and integrase (11). The 8E5 cell line expresses all the other HIV structural and regulatory proteins and secretes noninfectious particles into the medium. Because 8E5 cells are $\mathrm{CD}^{-}$, they are refractory to superinfection by HIV.

The $8 \mathrm{E} 5$ cell line is ideally suited for investigations of HIV recombination in tissue culture. Since the defective integrated provirus it harbors directs synthesis of viral mRNA, proteins, and noninfectious viral particles, the introduction of other mutated HIV proviral DNAs into 8E5 cells could provide the recombinational partners necessary to generate replication-competent progeny. As shown in Fig. 1, three different replication-deficient proviruses were constructed by mutagenizing the infectious molecular clone pNL4-3 (2). The pNLtat plasmid contains back-to-back termination codons at residues 11 and 12 of the tat open reading frame (ORF). Likewise, pNLint contains tandem termination codons at amino acids 14 and 15 in the portion of the pol ORF that encodes the HIV endonuclease-integrase. After the transfection of nonlymphoid cells, this clone directs the synthesis of noninfectious viral particles (as monitored by $\mathrm{RT}$ production) which are unable to initiate an infection in

\footnotetext{
* Corresponding author.
}

$\mathrm{CD}^{+}$lymphocytes (R. Willey, unpublished observations). The third plasmid, pNLenv-1, is a derivative of the infectious HIV-cloned provirus containing a 1,289-base-pair deletion (between nucleotides 6379 and 7668) in the env gene. Following transfection of SW480 colon carcinoma cells (2), pNLenv-1 directs the synthesis of normal amounts (compared with the parental plasmid) of gag and pol gene products but neither of the envelope glycoproteins (K. Strebel, unpublished observations). None of the three HIV mutant clones produced infectious particles following transfection of $\mathrm{CD}^{+}{ }^{+} \mathrm{A} 3.01$ cells.

The generation of HIV recombinant particles was attempted by transfecting $8 \mathrm{E} 5$ cells with each of the three mutant proviral DNAs. At $48 \mathrm{~h}$ following transfection, 1-ml

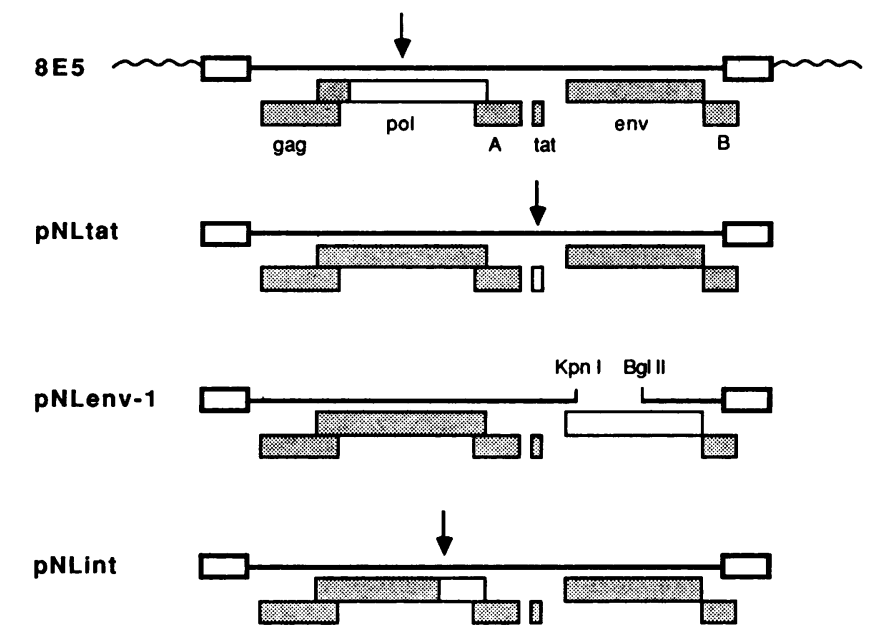

FIG. 1. Location of mutations in the HIV proviral DNAs used in transfection experiments. The arrows indicate the position of point mutations which cause premature termination. 8E5 cells (10) contain a single defective HIV provirus missing a single nucleotide within the RT domain of the pol gene at position 3492 (11) which precludes the synthesis of RT and integrase proteins. Plasmids pNLtat, pNLenv-1, and pNLint are derivatives of the infectious HIV clone pNL4-3 (2), and all contain mutagenized HIV proviral sequences. Back-to-back termination codons were introduced at positions 5898 (pNLtat) and 4307 (pNLint) by site-directed mutagenesis (31). The pNLenv-1 plasmid contains a 1,289-base-pair deletion between the KpnI site at position 6379 and the BglII site at position 7668 . The HIV ORFs affected by the mutations are indicated by the open rectangles. 
A

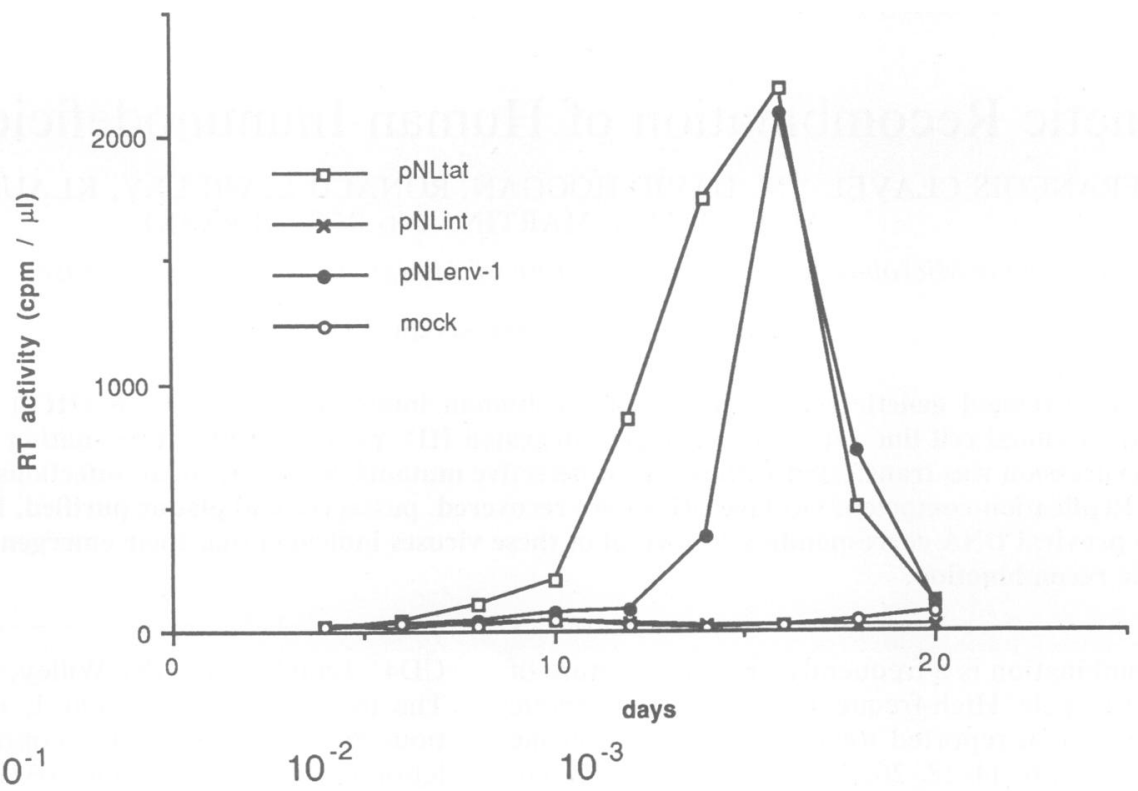

B

pNLenv-1
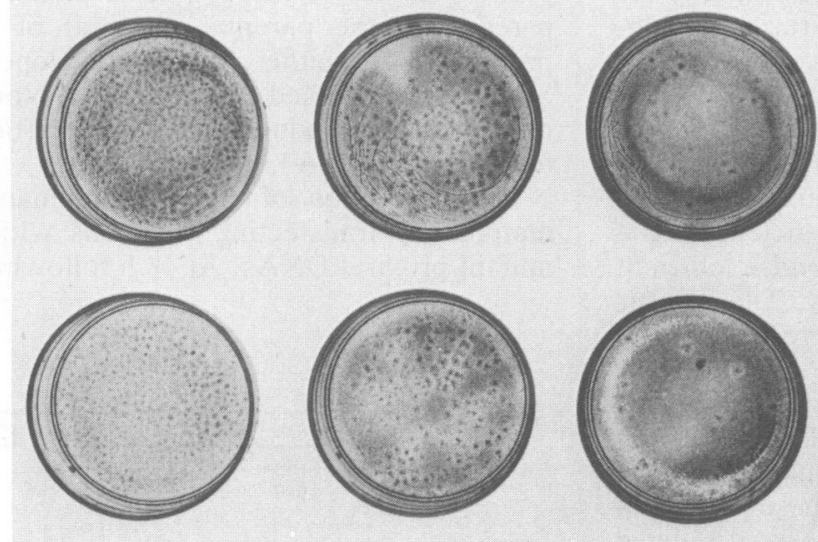

$p$ NLtat

$10^{-2}$
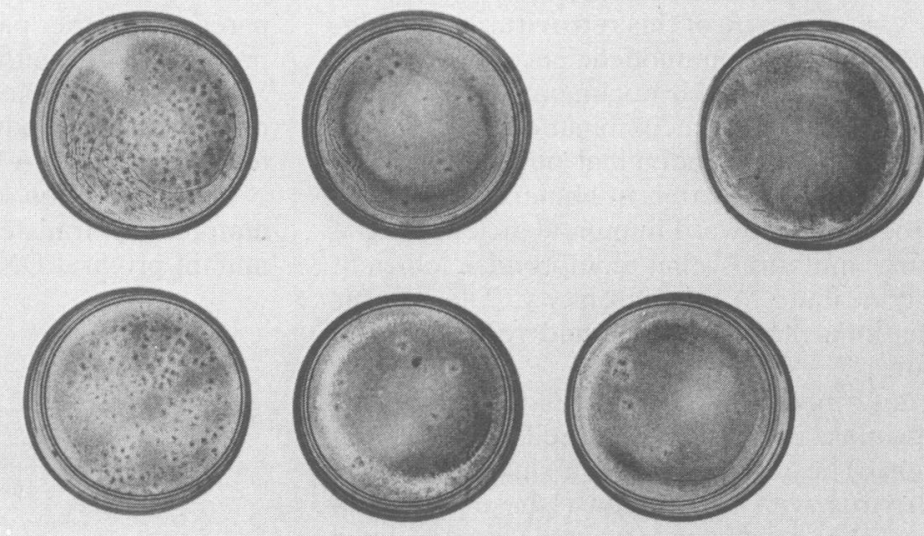

$10^{-5}$

FIG. 2. Infectivity of recombinant viruses generated following transfection of 8E5 cells with defective molecular clones of HIV. Approximately $2 \times 10^{6} 8 \mathrm{E} 5$ cells were transfected with $10 \mu \mathrm{g}$ of plasmid DNA by the DEAE-dextran technique (7). Two days later, a 1-ml sample of cell-free supernatant was collected and incubated with $2 \times 10^{6} \mathrm{~A} 3.01$ cells (final volume, 2 ml); RT production was monitored as previously described (29). Virus produced during a single passage in A3.01 cells was plaqued on MT4 cells (a gift from Jay Levy) as previously reported (12). (A) RT activity in the supernatants of A3.01 cells infected with cell-free supernatants harvested from 8E5 cells that had been transfected with the indicated plasmids. (B) Plaques generated on MT4 cell monolayers by A3.01-passaged recombinant HIV. In each case, cell-free medium from 8E5 cells (undiluted) or 8E5 cells transfected with the indicated plasmids was used for the infection of A3.01 cells.

samples of the tissue culture medium were removed and used for infection of A3.01 cells; these supernatants contained no detectable RT activity. A spreading infection was detected in the cultures exposed to medium from the pNLtat and $\mathrm{pNLenv-1}$ transfections; no infectious virus was detected in the A3.01 cells incubated with the pNLint supernatant (Fig. 2A). In several experiments of this type, progeny virions resulting from infection with pNLtat medium were initially detected 3 to 4 days before virus appeared in cultures infected with the pNLenv-1 supernatants.

Infectious virus present in the medium of pNLtat- and pNLenv-1-transfected cells was also evaluated by the MT4 plaque assay (12). Compared with supernatants from 8E5 cells transfected with the wild-type pNL4-3 plasmid, the media from pNLtat and pNLenv-1 transfections of $8 \mathrm{E} 5$ cells contained 15 and $1.5 \%$ PFU, respectively. In a separate experiment, virus generated from the pNLtat- and the pNLenv-1-infected A3.01 cultures were plated on MT4 cells. Approximately 10-fold-more pNLtat- than pNLenv-1-derived virus was produced in the infected A3.01 cells (Fig. 2B). Of note was the consistently smaller plaque size observed with the pNLenv-1 derivative. Several individual plaques of both putative HIV recombinants were isolated and passaged twice in A3.01 cells. In all cases, the synthesis of progeny particles was associated with syncytium formation and cell killing.

The mutagenized pNL4-3 provirus used in the transfection experiments and the defective 8E5 proviral DNA have restriction maps that can readily distinguish one virus from another (Fig. 3A). These differences provided a convenient 

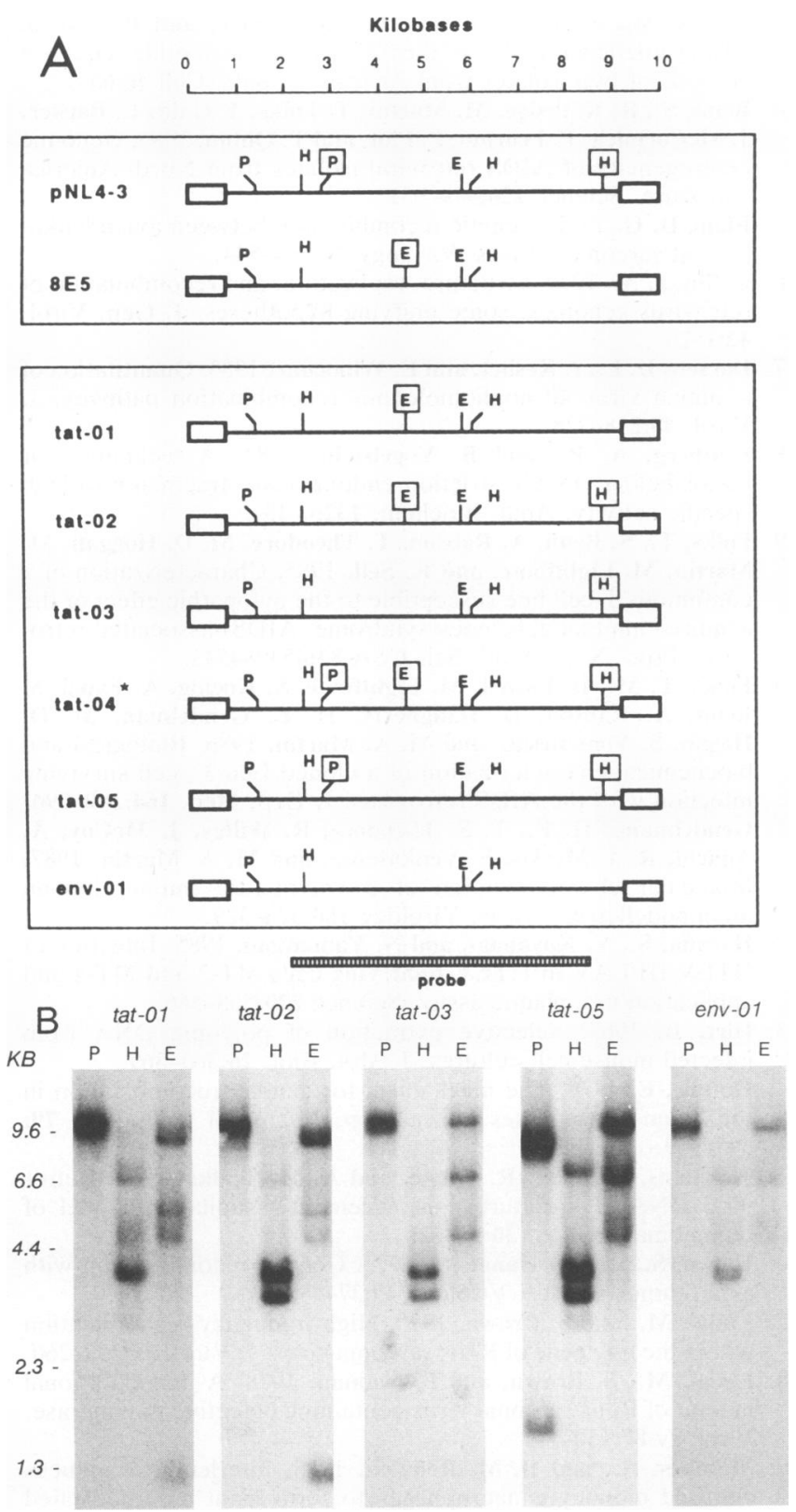

FIG. 3. Restriction mapping of HIV proviral DNAs present in cells infected with plaque-purified recombinant viruses. Individual plaques generated by HIV recombinants on MT4 cell monolayers were removed by gentle aspiration with a $1-\mathrm{ml}$ pipette and suspended in $1 \mathrm{ml}$ of RPMI 1640 medium. A3.01 cells $\left(2 \times 10^{6}\right)$ were added to a final volume of $2 \mathrm{ml}$. At the peak of virus production approximately 7 days after the infection, 1-ml samples of cell-free supernatants were harvested and used to infect $15-\mathrm{ml}$ cultures of A3.01 cells $\left(10^{6}\right.$ cells per $\left.\mathrm{ml}\right)$. When syncytia and cell lysis were initially noted (5 to 7 days following infection), cellular DNA was extracted, fractionated by the method of Hirt (13), and digested with Pstl, HincII, or EcoRI. A $10-\mu \mathrm{g}$ sample of each of the digested DNAs was electrophoresed on a $0.7 \%$ agarose gel and transferred to a nylon membrane. Hybridization was performed as described previously (4) with $10^{6} \mathrm{cpm}$ of the pBENN 6 insert per $\mathrm{ml}(9)$. This segment contains $6.5 \mathrm{~kb}$ of the HIV-1 provirus from nucleotides 1712 to 8188 and was ${ }^{32} \mathrm{P}$ labeled by the random hexamer method (8). (A) The restriction maps of the parental pNL4-3 and 8E5 proviruses (upper box) have been previously published (11). Restriction maps were constructed for the proviral DNAs present in A3.01 cells infected with plaque-purified viral recombinants derived from approach for the demonstration of genetic recombination between pairs of defective HIVs. Hirt supernatant DNA was prepared from A3.01 cultures infected with plaque-purified putative viral recombinants, digested with EcoRI, HincII, and PstI, and analyzed by Southern blot hybridization with the pBENN 6 probe (9), which contains a 6.5-kilobase (kb) segment spanning the gag, pol, and env genes of HIV. The result of this analysis is presented in Fig. 3B. Four of the plaque-purified viruses (tat-02, tat-03, tat-04, and env-01) synthesized proviral DNAs with restriction maps unlike either parent. Instead, they exhibited different arrangements of the parental polymorphic restriction sites, strongly suggesting that they arose by a recombinational process. Two (tat-01 and tat-05) gave restriction patterns that were indistinguishable from parental proviruses. Analyses with restriction enzymes which gave a pattern that did not differ in the parental proviruses (such as HindIII), showed that no modification of this pattern occurred in any of the recombinants (data not shown), suggesting that the differences between progeny viruses were not the consequence of point mutations. An analysis of these results strongly suggests that a complex process involving multiple crossing-over events occurred during the generation of infectious viruses. For example, to generate replication-competent tat- 03 particles, at least one exchange took place between the HincII site at position 8800 and the tat ORF to repair the tat mutation, and at least a double crossing-over occurred between the tat ORF and the HincIII site at position 2555 to restore RT function. For those progeny viruses whose restriction pattern did not differ from that of the parental proviruses, the exchanges of genetic material that are likely to have occurred spared the analyzed polymorphic restriction sites. In general, the pattern of recombination observed is consistent with the exchange of short segments of genetic information from each partner as has been reported for recombination among avian and murine retroviruses $(1,6,30)$.

Although it is possible that the infectious virions emerging from the transfected $8 \mathrm{E} 5$ cells in our experiments arose as a consequence of direct homologous recombination between the transfected and integrated proviral DNAs, this seems very unlikely in view of the very high frequency (15 to $20 \%$ yields of infectious pNLtat virus compared with wild type) of recombination. In mammalian systems, in which recombination between transfected and genomic DNAs has been carefully studied, extremely low levels of exchange have been observed $(22,25)$. In addition, our failure to recover infectious virus following the transfection of the pNLint plasmid would not be consistent with a direct recombination between transfected and genomic proviral sequences. Since neither pNLint nor $8 \mathrm{E} 5$ proviruses are capable of synthesiz-

pNLtat (tat-01, tat-02, tat-03, tat-04, and tat-05)- or pNLenv-1 (env-01)-transfected 8E5 cells (lower box). Restriction enzymes used include PstI (P), HincII (H), and EcoRI (E). The asterisk indicates that an accompanying Southern blot is not presented. (B) Southern blots of DNA extracted from A3.01 cells infected with the indicated plaque-purified viruses. The polymorphic PstI site at position 2800 , unique to the pNL4-3 provirus, generates a $1.8-\mathrm{kb}$ reactive fragment (as seen in tat-05). The HincII site at position 8800 (also derived from pNL4-3) generates a 3.0-kb cleavage product seen in tat-02, tat-03, and tat-05 digests. In some Hincll digests, the $6.5-\mathrm{kb}$ product represents a partial cleavage product. The unique EcoRI site at position 4684 of the $8 \mathrm{E} 5$ proviral DNA gives rise to a characteristic $1.1-\mathrm{kb}$ fragment, seen in tat-01 and tat-02 digests. The other fragments observed in EcoRI digests are generated by cleavage of either circular or linear unintegrated proviral DNA molecules. 
ing the endonuclease-integrase, the absence of pNLint recombinants is compatible with the requirement to form phenotypically complete and probably heterozygotic virions as intermediates in the recombination process. Several mechanisms have been proposed for retroviral recombination $(6,14,15,27)$. Most of them involve the reverse transcription of heterozygotic RNA dimers in conjunction with a second cycle of replication that follows the production of phenotypically mixed particles by dually infected cells. These mechanisms are consistent with our findings and with the high frequencies of intragenomic and intragenic exchanges observed in other retroviral systems.

Analyses of proviruses corresponding to different HIV-1 isolates reveal extensive variability at the nucleotide level consisting of point mutations, deletions, insertions, and repetitions $(3,23,28)$. Although a majority of these alterations may be due to erroneous reverse transcription or DNA-dependent RNA polymerization, further variability may result from the high levels of genetic recombination attending retrovirus replication. Indeed, phylogenetic analyses (20) of HIV-1 genomes indicate that some individual viral isolates contain both "ancient" and "contemporary" sequences and suggest that genetic recombination could have played a role in their generation.

Our findings could also provide an explanation for the slow disease progression characteristic of HIV infection. Following the initial exposure to the virus, a strong immunologic response, together with the cytopathic properties of the virus itself, should eliminate virtually all virus-producing cells. A large proportion of survivor cells will contain either nonfunctional proviruses or defective copies of proviral DNA capable of synthesizing some of the viral proteins. As we observed for $8 \mathrm{E} 5$ in this study, genetic recombination could generate replication-competent viruses from such a collection of defective proviral sequences. A number of scenarios incorporating unique features of the HIV life cycle can be envisaged in which recombinational mechanisms could give rise to infectious virus. Two defective HIV proviruses containing mutations affecting different genes might be brought together in the same cell following receptor-mediated cell-to-cell fusion. Such fusion of HIV-infected cells could also be achieved in the absence of gp120-CD4 interaction; it is well known, for example, that activated $\mathrm{T}$ cells can produce factors that induce monocytes-macrophages to form multinucleated cells $(19,21)$, thereby creating a milieu in which recombination might occur. The resulting viruses could in turn spread to noninfected cells, reinitiate the cycle, and ultimately involve the entire immune system. Repeated recombinations could also lead to the emergence of infectious particles with novel pathogenic potential, as recently reported (24). This could herald the end of the asymptomatic phase of an HIV infection and result in disease progression.

We thank Tom Folks and S. Venkatesan for their interest in this work.

\section{LITERATURE CITED}

1. Aaronson, S. A., and M. Barbacid. 1980. Viral genes involved in leukemogenesis. I. Generation of recombinants between oncogenic and non-oncogenic mouse type-C viruses in tissue culture. J. Exp. Med. 151:467-480.

2. Adachi, A., H. E. Gendelmann, S. Koenig, T. Folks, R. Willey, A. Rabson, and M. A. Martin. 1986. Production of acquired immunodeficiency syndrome-associated retrovirus in human and non-human cells transfected with an infectious molecular clone. J. Virol. 59:284-291.
3. Alizon, M., S. Wain-Hobson, L. Montagnier, and P. Sonigo. 1986. Genetic variability of the AIDS virus: nucleotide sequence analysis of two isolates from African patients. Cell 46:64-73.

4. Benn, S., R. Rutledge, M. Martin, T. Folks, J. Gold, L. Barker, J. McCormick, P. Feorino, P. Piot, and T. Quinn. 1985. Genomic heterogeneity of AIDS retroviral isolates from North America and Zaire. Science 220:949-951.

5. Blair, D. G. 1977. Genetic recombination between avian leukosis and sarcoma viruses. Virology 77:534-544.

6. Coffin, J. M. 1979. Structure, replication, and recombination of retrovirus genomes: some unifying hypotheses. J. Gen. Virol. 42:1-26.

7. Dorsett, D. L., I. Keshet, and E. Winocour. 1983. Quantitation of a simian virus 40 nonhomologous recombination pathway. $\mathrm{J}$. Virol. 48:218-228.

8. Feinberg, A. P., and B. Vogelstein. 1983. A technique for radiolabeling DNA restriction endonuclease fragments to high specific activity. Anal. Biochem. 132:6-13.

9. Folks, T., S. Benn, A. Rabson, T. Theodore, M. D. Hoggan, M. Martin, M. Lightfoote, and K. Sell. 1985. Characterization of a continuous T-cell line susceptible to the cytopathic effect of the acquired immunodeficiency syndrome (AIDS)-associated retrovirus. Proc. Natl. Acad. Sci. USA 83:4539-4543.

10. Folks, T. M., D. Powell, M. Lightfoote, S. Koenig, A. Fauci, S. Benn, A. Rabson, D. Daugherty, H. E. Gendelman, M. D. Hogan, S. Venkatesan, and M. A. Martin. 1986. Biological and biochemical characterization of a cloned Leu- $3^{-}$cell surviving infection with the AIDS retrovirus. J. Exp. Med. 164:280-290.

11. Gendelmann, H. E., T. S. Theodore, R. Willey, J. McCoy, A. Adachi, R. J. Mervis, S. Venkatesan, and M. A. Martin. 1987. Molecular characterization of a polymerase mutant human immunodeficiency virus. Virology 160:323-329.

12. Harada, S., Y. Koyanagi, and N. Yamamoto. 1985. Infection of HTLV-III/LAV in HTLV-I-carrying cells MT-2 and MT-4 and application in a plaque assay. Science 229:568-566.

13. Hirt, B. 1967. Selective extraction of polyoma DNA from infected mouse cell cultures. J. Mol. Biol. 26:365-369.

14. Hunter, E. 1978. The mechanism for genetic recombination in the avian retroviruses. Curr. Top. Microbiol. Immunol. 79: 295-309.

15. Junghans, R. P., L. R. Boone, and A. M. Skalka. 1982. Retroviral DNA $H$ structures: displacement-assimilation model of recombination. Cell 30:53-62.

16. Kawai, S., and H. Hanafusa. 1972. Genetic recombination with avian tumor viruses. Virology 49:37-44.

17. Linial, M., and S. Brown. 1979. High-frequency recombination within the gag gene of Rous sarcoma virus. J. Virol. 31:257-260.

18. Linial, M., S. Brown, and P. Neiman. 1978. A nonconditional mutant of Rous sarcoma virus containing defective polymerase. Virology 87:130-141.

19. McInnes, A., and D. M. Rennick. 1988. Interleukin 4 induces cultured monocyte/macrophages to form giant multinucleated cells. J. Exp. Med. 167:598-611.

20. Myers, G., A. B. Rabson, S. F. Josephs, T. F. Smith, and F. Wong-Staal (ed.). 1987. Human retroviruses and AIDS, p. III-32. Los Alamos National Laboratory, Los Alamos, New Mexico.

21. Nagasawa, H., C. Miyaura, E. Abe, T. Suda, M. Horiguchi, and T. Suda. 1986. Fusion and activation of human alveolar macrophages induced by recombinant interferon-gamma and their suppression by dexamethasone. Am. Rev. Respir. Dis. 136: 916-921.

22. Smithies, O., R. G. Gregg, S. S. Boggs, M. A. Koralewski, andR. S. Kucherlapati. 1985. Insertion of DNA sequences into the human chromosomal $\beta$-globin locus by homologous recombination. Nature (London) 317:230-234.

23. Starcich, B. R., B. H. Hahn, G. M. Shaw, P. D. McNeely, S. Modrow, H. Wolf, E. S. Parks, W. P. Parks, S. F. Josephs, R. C. Gallo, and F. Wong-Staal. 1986. Identification and characterization of conserved and variable regions in the envelope gene of HTLV-III/LAV, the retrovirus of AIDS. Cell 45:637-648.

24. Tersmette, M., R. E. Y. de Goede, B. J. M. Al, I. N. Winkel, R. A. Gruters, H. T. Cuypers, H. G. Huisman, and F. Miedema. 
1988. Differential syncytium-inducing capacity of human immunodeficiency virus isolates: frequent detection of syncytiuminducing isolates in patients with acquired immunodeficiency syndrome (AIDS) and AIDS-related complex. J. Virol. 62: 2026-2030.

25. Thomas, K. R., K. R. Folger, and M. R. Capecchi. 1986. High frequency targeting of genes to specific sites in the mammalian genome. Cell 44:419-428.

26. Vogt, P. K. 1971. Genetically stable reassortment of markers during mixed infection with avian tumor viruses. Virology 46:947-952.

27. Weiss, R. A., W. S. Mason, and P. K. Vogt. 1973. Genetic recombinants and heterozygotes derived from endogenous and exogenous avian RNA tumor viruses. Virology 52:535-552.

28. Willey, R. L., R. A. Rutledge, S. Dias, T. Folks, T. Theodore, C. E. Buckler, and M. A. Martin. 1986. Identification of con- served and divergent domains within the envelope gene of the acquired immunodeficiency syndrome retrovirus. Proc. Natl. Acad. Sci. USA 83:5038-5042.

29. Willey, R. L., D. H. Smith, L. A. Lasky, T. S. Theodore, P. L. Earl, B. Moss, D. J. Capon, and M. A. Martin. 1988. In vitro mutagenesis identifies a region within the envelope gene of the human immunodeficiency virus that is critical for infectivity. J. Virol. 62:139-147.

30. Wyke, J. A., and J. A. Beamand. 1979. Genetic recombination in Rous sarcoma virus: the genesis of recombinants and lack of evidence for linkage between $\mathrm{pol}$, env, and $s r c$ genes in three factor crosses. J. Gen. Virol. 43:349-364.

31. Zoller, M. J., and M. Smith. 1984. Oligonucleotide-directed mutagenesis: a simple method using two oligonucleotide primers and a single-stranded DNA template. DNA 3:379-388. 